\section{Besonderheiten der Behandlung von Diabetikern im Alter}

In Deutschland erreicht die Lebenszeitprävalenz des Typ-2-Diabetes mellitus (T2D) stetig ansteigend in der 8. bzw. 9. bis 10. Lebensdekade Maximalwerte von $22 \%$ [1] bzw. 25\% [2]. Ab der 8. Lebensdekade ist dabei auch der zwischen der 5. und 7. Lebensdekade zu beobachtende, nahezu 2fache Prävalenzunterschied zwischen Frauen und Männern aufgehoben [1]. Für die Häufigkeit des Diabetes mellitus Typ 1 (T1D) in Deutschland wird ab der 9. Lebensdekade die frappant hohe Zahl von $0,5 \%$ genannt [2]. All diese Informationen stammen aus Erhebungen im Zeitraum von 2008 bis 2011 und basieren entweder auf dem sog. DEGS1-Survey des Robert Koch-Instituts [1] oder auf Auswertungen des sog. DIMDI-Datensatzes [2]. Sie gelten als die aktuellsten verfügbaren epidemiologischen Daten zu Altersdiabetes in Deutschland.

Der Begriff „Altersdiabetologie“ im Sinne einer gesonderten Betrachtung vornehmlich des T2D ab einem Alter von 70 Jahren leitet sich nicht aus herausragenden altersabhängigen pathophysiologischen Eigenheiten des Glukosestoffwechsels ab; diese sind eher marginal [3]. „Altersdiabetologie“ bedeutet vielmehr die Einbeziehung altersmedizinischen Spezialwissens über die funktionale Gesundheit zum Zwecke einer abwägend-individuellen Diabetesbehandlung im Kontext geriatrischer Multimorbidität und Polypharmazie.

Dieser Auffassung tragen zwischenzeitlich sowohl die American Diabetes Association (ADA) mit ihren im Jahresrhythmus erscheinenden Standards of Medical Care in Diabetes [4], als auch die S2k-Leitlinie der Deutschen Diabetes Gesellschaft (DDG) zur Diagnostik, Therapie und Verlaufskontrolle des Diabetes mellitus im Alter Rechnung [5].

Aus Meta- und Subgruppenanalysen der großen „Megatrials“ ACCORD, ADVANCE und VADT sowie aus altersdiabetologischen Beobachtungsstudien erwuchs die Erkenntnis, dass die Auswirkung/Relevanz Diabetes-assoziierter kardiovaskulärer Risikofaktoren (Hyperglykämie, Hypertonie, Dyslipidämie, Mikroalbuminurie, Rauchen) im hohen Lebensalter abnimmt [6] Mehr noch: Eine zu straffe Blutzuckereinstellung ist gerade im Alter aufgrund der damit einhergehenden Hypoglykämiegefahr (Blutzucker $<72 \mathrm{mg} / \mathrm{dl}$ [4 mmol/l] [7]) eher schädlich als nutzbringend, wobei insbesondere die demenzfördernde Wirkung bereits einzelner Hypoglykämieepisoden hervorgehoben werden muss [8].

Als Richtwerte für eine adäquate Blutzuckereinstellung eines typisch geriatrischen Patienten mit leicht bis mittelgradig eingeschränkten Funktionsressourcen können gelten: HbA1c < 7,5\% (58,5 mmol/ mmol), Nüchtern-Blutzucker 90-130 mg/ dl (5-7,2 mmol/l), Blutzucker zur Nacht 90-180 mg/dl (5-8,3 mmol/l) [4].

Bei der medikamentösen Antidiabetestherapie des T2D gilt auch im fortgeschrittenen Lebensalter Metformin als das FirstLine-Medikament [9]. Allerdings darf Metformin bei einer glomerulären Filtrationsrate (GFR) von $<45 \mathrm{ml} / \mathrm{min}$ nicht neu angesetzt bzw. muss unterhalb einer GFR $<30 \mathrm{ml} / \mathrm{min}$ abgesetzt werden. Bei einer GFR zwischen 30 und $59 \mathrm{ml} / \mathrm{min}$ ist ferner eine Tagesdosis von $\leq 1000 \mathrm{mg}$ zu beachten.

Vor dem Hintergrund bestmöglicher Hypoglykämievermeidung und der im Alter abnehmenden Nierenfunktion kommt nach dem Metformin der Substanzklasse der DPP-IV-Inhibitoren (Gliptine) eine differenzialtherapeutische Bedeutung in der Altersdiabetologie zu [10]. In der aktuellen sog. FORTA-Liste aus dem Jahre 2018 werden die DPP-IV-Inhibitoren sogar als einzige antidiabetische Substanzklasse der Kategorie A aufgeführt und damit differenzialtherapeutisch favorisiert [11].

Der Einsatz von Insulin-Sekretagoga (Sulfonylharnstoffe, Glinide) ist in der Altersdiabetologie wegen der besonderen Gefahr schwerwiegender und anhaltender Hypoglykämien sowie aufgrund multipler Arzneimittelinteraktionen zu vermeiden $[3,12]$.

In der Altersdiabetologie bis dato (zu) wenig etabliert sind die SGLT-2-Inhibitoren
(Gliflozine) und die GLP-1-Rezeptor-Analoga, obwohl gerade diese beiden Substanzklassen im Rahmen aktueller Studien mit dem daraus resultierenden differenzialtherapeutischen Paradigmenwechsel des sog. EASD/ADA-Konsensus aus dem Jahre 2018 [13] generell als (nach Metformin) zu bevorzugende Therapeutika in der Diabetologie gelten. Die bisherigen geriatrischen Vorbehalte aufgrund urogenitaler Infektionen (Gliflozine) oder der anorexogenen Wirkung (GLP-1-Rezeptor-Analoga) sind vor diesem Hintergrund neu abzuwägen.

Was den Stellenwert der Insulintherapie beim T2D im Alter anbelangt, so gilt eindeutig die Regel: Keep it simple [4]. Folgerichtig sollte in der Geriatrie die einmal tägliche Gabe einer fixen Menge an Insulin, wie z.B. im Rahmen der sog. BOT (basal unterstützte orale Antidiabetestherapie), das Ziel sein. Komplexe, am präprandialen Blutzucker ausgerichtete und mahlzeitenbezogene Insulingaben unter Berücksichtigung von Insulin:Kohlenhydrat-Quotienten und Korrekturregeln gehen an der Praxis vorbei und bergen die Gefahr der Übertherapie im Sinne einer „Pseudo-ICT“ ohne Zusatznutzen, aber mit hoher Komplikationsgefahr.

Interessenkonflikt

Die Autoren geben an, dass kein Interessenkonflikt besteht.

Autorinnen/Autoren

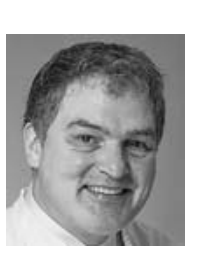

Cornelius Bollheimer

Univ.-Prof. Dr. med., Lehrstuhl für Altersmedizin der Uniklinik RWTH Aachen mit Klinik für Innere Medizin und Geriatrie am Franziskushospital Aachen

\section{Korrespondenzadresse}

Univ.-Prof. Dr. med. Cornelius Bollheimer Lehrstuhl für Altersmedizin der Uniklinik RWTH Aachen

Klinik für Innere Medizin und Geriatrie Franziskushospital Aachen

Morillenhang 27

52074 Aachen

cbollheimer@ukaachen.de 


\section{Literatur}

[1] Heidemann C, Du Y, Schubert I et al. Prävalenz und zeitliche Entwicklung des bekannten Diabetes mellitus. Bundesgesundheitsbl 2013; 56: 668-677

[2] Tamayo T, Brinks R, Hoyer A et al. Prävalenz und Inzidenz von Diabetes mellitus in Deutschland. Dtsch Arztebl 2016; 113: 177-182

[3] Laurentius T, Freitag M, Eitner J et al. Glukosestoffwechsel im fortgeschrittenen Lebensalter. Internist (Berlin) 2019; 60: 133-140

[4] American Diabetes Association. 12. Older Adults: Standards of Medical Care in Diabetes-2019. Diabetes Care 2019; 42: S139S147

[5] Deutsche Diabetes Gesellschaft. S2 k-Leitlinie Diagnostik, Therapie und Verlaufskontrolle des Diabetes mellitus im Alter. AWMFRegisternummer: 057-017 (2018). Im Internet: https://www.awmf.org/uploads/tx_
szleitlinien/057-017I_S2k_Diabetes_mellitus_ im_Alter_2018-09.pdf; Stand: 14.08.2019

[6] Rawshani A, Rawshani A, Franzén S et al. Risk Factors, Mortality, and Cardiovascular Outcomes in Patients with Type 2 Diabetes. N Engl J Med 2018; 379: 633-644

[7] Sinclair A, Morley JE, Rodriguez-Mañas L et al. Diabetes mellitus in older people: position statement on behalf of the International Association of Gerontology and Geriatrics (IAGG), the European Diabetes Working Party for Older People (EDWPOP), and the International Task Force of Experts in Diabetes. J Am Med Dir Assoc 2012; 13: 497-502

[8] Mattishent K, Loke YK. Bi-directional interaction between hypoglycaemia and cognitive impairment in elderly patients treated with glucose-lowering agents: a systematic review and meta-analysis. Diabetes Obes Metab 2016; 18: 135-141

[9] Du YF, Ou HY, Beverly EA et al. Achieving glycemic control in elderly patients with type 2 diabetes: a critical comparison of current options. Clin Interv Aging. 2014; 9: 1963-1980

[10] Strain WD, Lukashevich V2, Kothny W et al. Individualised treatment targets for elderly patients with type 2 diabetes using vildagliptin add-on or lone therapy (INTERVAL): a 24 week, randomised, double-blind, placebocontrolled study. Lancet 2013; 382: 409-416

[11] Pazan F et al. The FORTA (Fit fOR The Aged) List 2018: Third Version of a Validated Clinical Tool for Improved Drug Treatment in Older People. Drugs Aging 2019; 36: 481-484

[12] Petri H. Analyse von CYP450-Wechselwirkungen - kleiner Aufwand, große Wirkung. Krankenhauspharmazie 2017; 38: 42

[13] Davis M], D’Alessio DA3, Fradkin J et al. Management of hyperglycaemia in type 2 diabetes, 2018. A consensus report by the American Diabetes Association (ADA) and the European Association for the Study of Diabetes (EASD). Diabetologia 2018; 61: 2461-2498 